\title{
Prescription de chlorhydrate de méthylphénidate : la vigilance s'impose
}

\section{Methylphenidate hydrochloride : cautiousness must be done}

Anne-Laure PELISSIER-ALICOT,

Service de Médecine Légale, Faculté de Médecine, 27, boulevard Jean Moulin - 13385 Marseille Cedex 5 Tél : + 330491324516 - Fax : +330491324512 - E-mail : apelissier@netcourrier.com

\section{$R E ́ S U M E ́$}

Le chlorhydrate de méthylphénidate (MPH) est un dérivé de la phénéthylanine classé comme stimulant du système nerveux central. Il agit essentiellement en augmentant la biodisponibilité de la dopanine au niveau de son récepteur. post-synaptique. Le MPH est commercialisé en France sous une forme à libération inmédiate (Ritaline ${ }^{\otimes}$ ) et sous deux formes à libération prolongée (Ritaline ${ }^{\circledast} L P$, Concerta ${ }^{\circledast} L P$ ). Ses indications sont (i) le traitement du trouble d'hyperactivité avec déficit de l'attention. (THADA) et (ii) le traitement de la narcolepsie avec ou sans cataplexie chez l'adulte et l'enfant de plus de six ans. Le MPH est inscrit sur la liste des stupéfiants et sa prescription doit être effectuée sur ordonnance sécurisée limitée à 28 jours. Il est également soumis à une prescription initiale hospitalière annuelle. Parmi ses effets secondaires figure le risque d'abus et de dépendance. Ces comportenents s'observent soit dans un contexte récréatif, en général chez des étudiants, soit chez des patients traités pour un THADA, et s'inscrivent souvent dans un contexte de poly'consommation. Il convient cependant de prendre en compte le fait que le THADA constitue en luimême un facteur de risque de toxicomanie. Sur le plan analytique, le MPH et son métabolite, l'acide ritalinique, sont identifiables et dosables dans le sang et l'urine par chromatographie en phase gazeuse couplée à la spectrométrie de masse, ainsi que par chromatographie en phase liquide couplée à la spectrométrie de masse ou à la masse tandem, cette denière technique étant actuellement retenue par la majorité des auteurs.

MOTS-CLÉS

Méthylphénidate, psychostimulant, addiction.

\section{SUMMARY}

Methylphenidate hydrochloride (MPH) is a phenetylamine derivate classified as central nervous system stimulant. It would enhance the bioavailability of dopamine at its postsynaptic receptor: It is available in France in immediate$\left(\right.$ Ritaline $^{\oplus}$ ) and sustained-release (Ritaline ${ }^{\otimes} L P$, Concerta $^{\oplus}$ $L P$ ) preparations. It is indicated for the treatment of attention deficit-hyperactivity disorder (ADHD) in children aged over 6 years, and for the treatment of narcolepsy with or without cataplexy in adults and children aged over 6 years. $M P H$ is on the restricted drugs list and the prescription is limited to a maximum of 28 days. Therefore, an initial hospital annual prescription is mandatory. Their adverse effects include the risk of misuse and dependence. Literature reports a recreational use among traditional-age students as well as abuse and dependence in patients with $A D H D$. These behaviours frequently occur in a polysubstance use context. Nevertheless, it must be noticed that ADHD is commonly cooccuming with other psychiatric disorders including substance abuse disorders. Identification and quantification of MPH and ritalinic acid, its main metabolite, could be done by gas chromatography-mass spectrometry, liquid chromatography-mass spectrometry, or liquid chromatography-tandem mass-spectrometry; which has become the method of choice today.

\section{KEY-WORDS}

Methylphenidate, psychostimulant, addiction. 


\section{Introduction}

Le chlorhydrate de méthylphénidate (MPH) est un stimulant du système nerveux central, commercialisé pour la première fois aux États-Unis en 1954 par les laboratoires CIBA-GEIGY ${ }^{\circledR}$ avec des indications larges dans le domaine de la neuropsychiatrie (1). Ses indications thérapeutiques ont été considérablement restreintes par la suite. En France, le MPH est actuellement disponible sous une forme à libération immédiate $\left(\right.$ Ritaline $\left.^{\oplus}\right)$ et sous deux formes à libération prolongée (Ritaline ${ }^{\oplus} \mathrm{LP}$, Concerta $\left.{ }^{\oplus} \mathrm{LP}\right)(2,3,4)$. Ses indications sont limitées au trouble déficitaire de l'attention avec hyperactivité (THADA) chez l'enfant de plus de six ans et l'adulte, et à la narcolepsie avec ou sans cataplexie. Ses règles de prescription sont très strictes, puisque le MPH est soumis à la réglementation sur les stupéfiants ainsi qu'à une prescription initiale hospitalière annuelle $(2,3,4)$. On assiste néanmoins à une augmentation significative du nombre de prescriptions ces dernières années, vraisemblablement sous la pression des parents et des enseignants (5). Ce phénomène doit inciter à la vigilance car le MPH semble doté d'un réel potentiel addictif. En effet, si les cas d'abus et de dépendance sont extrêmement rares aujourd'hui en France, probablement du fait des contraintes réglementaires précédemment citées, on observe, dans les pays présentant des règles de prescription moins strictes, en particulier aux États-Unis, le développement d'un usage récréatif chez les adolescents ainsi que d'authentiques cas de dépendance chez des sujets traités pour un THADA. Il nous a donc paru important de développer dans cet article les différents aspects concernant la prescription de MPH en insistant sur son potentiel toxicomanogène.

\section{Propriétés miques}

Le chlorhydrate de méthylphénidate ou chlorhydrate de méthyl $\alpha$-phényl- $\alpha$-(2-pipéridyl) acétate a pour formule moléculaire $\mathrm{C}_{14} \mathrm{H}_{19} \mathrm{NO}_{2} \mathrm{HCL}$ et masse moléculaire 269,8 . C'est un dérivé de la phénétylamine chimiquement proche de l'amphétamine (figure 1). Il se présente sous la forme d'une poudre blanche franchement soluble dans l'eau et le méthanol, soluble dans l'alcool, légèrement soluble dans le chloroforme et l'acétone. Son pKa est de 8,8 (6). Les formes commerciales se présentent sous la forme d'un mélange racémique des énantiomères d-thréo et l-thréo (d,l-threo-MPH) (figure 2). L'activité pharmacologique est principalement due à l'énantiomère $d$ - (7).

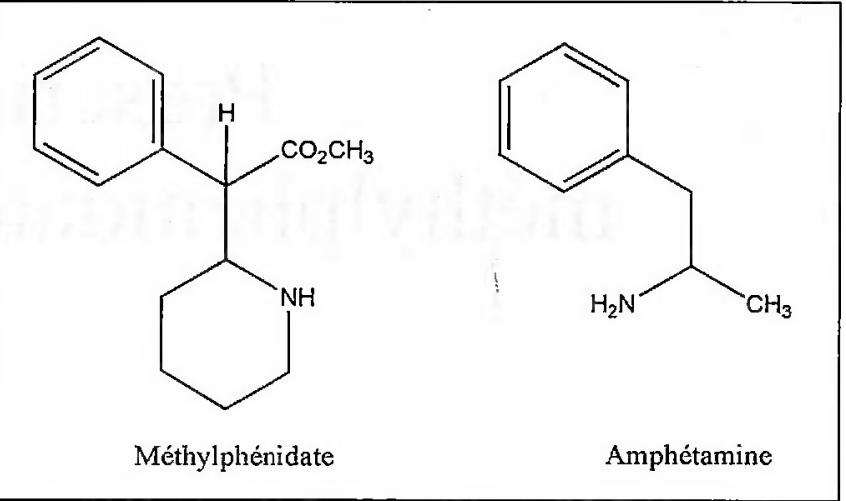

Figure 1 : Formules chimiques du méthylphénidate et de l'amphétamine, d'après Léonard et al. (6).

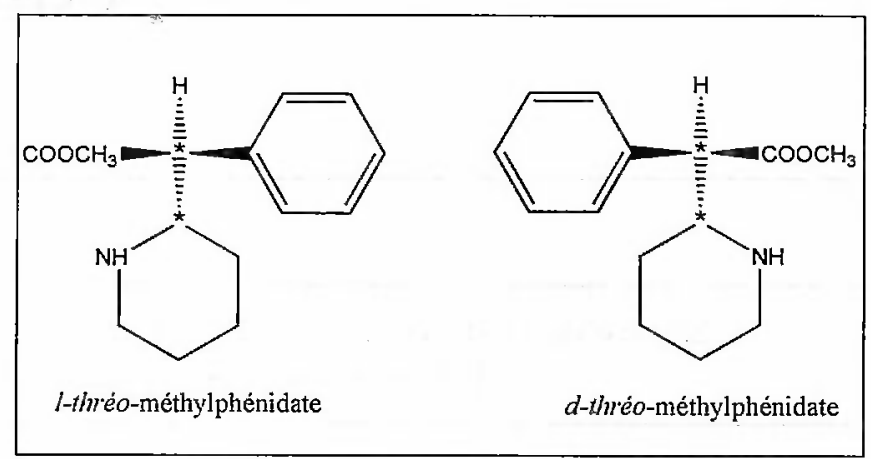

Figure 2 : Projections de Fisher des énantiomètres du méthylphénidate, d'après Sun et al. (11).

\section{Pharmacocinétique}

\section{Absorption}

Après administration orale d'une forme à libération immédiate, l'absorption intestinale du MPH est de l'ordre de $80 \%$. L'effet de premier passage hépatique est important, la biodisponibilité est variable, de l'ordre de 11 à $53 \%$ de la dose absorbée. Aux doses thérapeutiques, le Tmax est atteint en 1,5 à 2,5 h et le Cmax est de 6 à $15 \mathrm{ng} / \mathrm{ml}$ (8). L'absorption orale d'une forme à libération prolongée est caractérisée par un profil biphasique, avec un pic plasmatique initial de 3 à $17 \mathrm{ng} / \mathrm{ml}$ entre la $1^{\text {ire }}$ et la $4^{\text {ime }}$ heure après l'administration, suivi d'un second pic de 5 à $20 \mathrm{ng} / \mathrm{ml}$ entre la $3^{\text {ime }}$ et la $6^{\mathrm{am}}$ heure (9). Quelle que soit la formulation utilisée, l'absorption concomitante de nourriture accélère l'absorption mais ne modifie pas la quantité absorbée (10).

\section{Distribution}

Le MPH et ses métabolites se répartissent entre le plasma $(57 \%)$ et les érythrocytes (43\%). La liaison aux protéines plasmatiques est de l'ordre de $15 \%$ (11). Le MPH s'accumule rapidement dans les organes très vascularisés (foie, cœur, poumons, cerveau). Son volume apparent de distribution (Vd) est de $13 \mathrm{l} / \mathrm{kg}$ (6). 


\section{Biotransformation}

La biotransformation du MPH est rapide et extensive. Il est métabolisé à $80 \%$ par désestérification en acide $d$ - et $l$-ritalinique (acide alpha-phényl-2-pipéridine acétique) inactif (8) par la carboxylestérase hépatique CES1A1 (12) ainsi que par des estérases plasmatiques (13). Des métabolites hydroxylés inactifs, l'hydroxyméthylphénidate et l'acide hydroxyritalinique, sont également formés dans de faibles quantités (6). En présence d'alcool se forme un métabolite intermédiaire, l'éthylphénidate (14).

\section{Élimination}

La demi-vie d'élimination moyenne du MPH est de 2 heures. En 48 à $96 \mathrm{~h}$ après administration orale, 78 à $97 \%$ de la dose ingérée est excrétée dans les urines, et 1 à $3 \%$ dans les fécès sous forme de métabolites. Le principal métabolite urinaire est l'acide ritalinique, et l'on ne retrouve que de très faibles quantités $(1 \%)$ de MPH sous forme inchangée (15).

Enfin, la pharmacocinétique des deux énantiomères est légèrement différente. Après administration orale du mélange racémique, les concentrations plasmatiques du $d$-MPH sont systématiquement plus élevées que celles du $l-\mathrm{MPH}$, aussi bien pour la forme à libération immédiate que pour la forme à libération prolongée, alors qu'après administration intraveineuse, cet écart n'apparait que plus tardivement (16). Le volume apparent de distribution ainsi que l'aire sous la courbe des concentrations sont plus élevés pour l'énantiomère $d$ que pour l'énantiomère $l$-. Par contre, la clairance rénale des deux énantiomères est identique dans tous les cas. D'autre part, la carboxylestérase hépatique cataboliserait de manière plus rapide et plus extensive l'énantiomère $l$ - que l'énantiomère $d$-, alors que les estérases plasmatiques auraient une affinité inverse (13).

\section{Pharmacodynamie}

Le MPH est un agent sympathomimétique classé comme stimulant du système nerveux central de type noo-analeptique. Seul l'énantiomère $d$ - serait actif (17). Sa distribution dans le cerveau s'effectue préférentiellement au niveau des ganglions de la base du crâne, striatum en particulier, alors que celle de l'énantiomère $l$ - est homogène dans tout le tissu cérébral (17). Le mécanisme d'action du $d$-MPH n'est pas encore parfaitement élucidé. Il améliorerait la biodisponibilité de la dopamine au niveau de son récepteur post-synaptique (i) en augmentant sa libération dans la fente synaptique (18), (ii) en inhibant sa recapture au niveau des récepteurs DAT1 $(17,19)$, et (iii) en inhibant son catabolisme par le système des monoamine-oxydases (20). Ceci aurait pour conséquence d'augmenter la magnitude et la durée du signal dopaminergique dans le striatum (17). Il augmenterait également le débit sanguin dans les régions striatales et périventriculaires postérieures, et le diminuerait dans les régions sensorielles primaires, normalisant ainsi le flux sanguin cérébral chez les patients atteints de THADA, avec pour conséquence une augmentation des capacités de concentration et d'attention, ainsi qu'une diminution de l'hyperactivité motrice chez ces patients (15).

\section{Indications thérapeutiques}

En France, le laboratoire NOVARTIS ${ }^{\circledR}$ commercialise $^{2}$ le MPH sous forme de comprimés sécables à libération immédiate dosés à $10 \mathrm{mg}$ (Ritaline ${ }^{\circledast}$ ) depuis 1995, et sous forme de gélules à libération prolongée dosées à 20, 30 ou $40 \mathrm{mg}$ (Ritaline ${ }^{\oplus}$ LP) depuis $2003(2,3)$. Le laboratoire JANSSEN-CILAG ${ }^{\oplus}$ commercialise également depuis 2003 le MPH sous forme de comprimés à libération prolongée dosés à 18 et $36 \mathrm{mg}$ (Concerta ${ }^{\circledR}$ LP) (4). Les indications de ces différentes formes sont les suivantes :

- Trouble déficitaire de l'attention avec hyperactivité (THADA) chez l'enfant de plus de six ans, sans limite supérieure d'âge: la prescription est basée sur un diagnostic clinique établi sur l'évaluation par plusieurs intervenants (parents, éducateurs, médecins) de l'intensité et du caractère invalidant des troubles de l'attention, de l'impulsivité et de l'hyperactivité de l'enfant, et confirmé par un examen neuropsychologique (2). Le traitement est généralement initié avec la forme à libération immédiate à $10 \mathrm{mg}$, avec une posologie progressive, sans dépasser $1 \mathrm{mg} / \mathrm{kg} / \mathrm{j}$, jusqu'à une dose maximale de $60 \mathrm{mg} / \mathrm{j}$ en 2 à 3 prises quotidiennes afin d'obtenir des taux plasmatiques stables durant les heures d'apprentissage scolaire. Le relais à une forme LP est effectué dès l'optimisation du traitement, afin d'améliorer le confort de l'enfant en supprimant les prises multiples dans la journée. Les formes LP sont administrées en 1 dose unique le matin. Le choix de la dose se base sur une table d'équivalence (tableau I) (3). Il convient de noter que le Concerta ${ }^{\circledast} \mathrm{LP}$ peut être prescrit dans le traitement du THADA chez des enfants et des adolescents naïfs au MPH (4).

Tableau I : Adaptation des posologies de Ritaline LP $^{\otimes}$ et de Concerta ${ }^{\otimes}$ LPlors du relais de la forme à libération inmédiate à une forme à libération prolongée $(3,4)$.

\begin{tabular}{|l|l|l|}
\hline Posologie de Ritaline $10 \mathrm{mg}$ & Posologie de Ritaline ${ }^{\complement} \mathrm{LP}$ & Posologie de Concerta ${ }^{\circ} \mathrm{LP}$ \\
\hline 15 à $20 \mathrm{mg}$ & $20 \mathrm{mg}$ en 1 prise le matin & $18 \mathrm{mg}$ en 1 prise le matin \\
\hline $30 \mathrm{mg}$ & $30 \mathrm{mg}$ en 1 prise le matin & $36 \mathrm{mg}$ en 1 prise le matin \\
\hline 40 à $60 \mathrm{mg}$ & $40 \mathrm{mg}$ en 1 prise le matin & $54 \mathrm{mg}$ en 1 prise le matin \\
\hline
\end{tabular}


- Narcolepsie avec ou sans cataplexie, en cas d'inefficacité du modafinil (Modiodal ${ }^{\top}$ ), chez l'adulte et l'enfant de plus de six ans : cette indication ne concerne que la forme à libération immédiate (Ritaline ${ }^{\circledR} 10 \mathrm{mg}$ ). Dans les formes typiques de narcolepsie avec cataplexie, le diagnostic est purement clinique. Dans les formes atypiques de narcolepsie, le diagnostic doit être confirmé par un enregistrement polygraphique du sommeil (EPS) de nuit ou sur $24 \mathrm{~h}$, et par un test itératif de latence d'endormissement (TILE), ces deux examens devant être réalisés par un centre d'exploration du sommeil. Chez l'enfant, la posologie est la même que pour le traitement du THADA. Chez l'adulte, le traitement débute à 10 à $15 \mathrm{mg} / \mathrm{j}$ avec une adaptation progressive jusqu'à atteindre une posologie optimale de 20 à $30 \mathrm{mg} / \mathrm{j}(2)$.

Dans le cadre du THADA, la Commission de la Transparence recommande (i) de suivre les patients en milieu hospitalier pendant les deux premiers mois afin de contrôler l'efficacité du traitement et la posologie, et (ii) d'arrêter le traitement pendant les week-ends et les périodes de vacances $(2,3)$. Dans le cadre de la narcolepsie, la Commission recommande de n'utiliser le MPH qu'après échec d'un traitement bien conduit par le modafinil (Modiodal ${ }^{\circledR}$ ) (2).

A l'étranger sont actuellement développées des préparations contenant à la fois une forme retard et une forme rapide (Ritaline ${ }^{\oplus}$-LA) afin de pallier un phénomène de tachyphylaxie parfois observé avec les formes retard classiques (21). Des systèmes transdermiques sont également en cours d'élaboration (22). Enfin, d'autres indications hors AMM ont été suggérées pour le MPH : démence sénile (23), maladie d'Alzheimer (24), asthénie et douleurs d'origine cancéreuse (25). Son utilité dans le cadre de la prise en charge du syndrome de l'X fragile est également évoquée (26).

\section{Règles de prescription}

Le MPH est inscrit sur le Tableau II des substances psychotropes selon la convention de Vienne sur les stupéfiants (27). En France, il est inscrit sur la liste des stupéfiants et sa prescription doit être effectuée sur ordonnance sécurisée limitée à 28 jours $(2,3,4)$. Il est également soumis à une prescription initiale hospitalière annuelle réservée aux spécialistes et/ou aux services spécialisés en neurologie, psychiatrie, pédopsychiatrie et pédiatrie. La prescription initiale hospitalière a une validité d'1 an. Dans les périodes intermédiaires, tout médecin peut renouveler cette prescription. La délivrance est effectuée par un pharmacien d'officine sur présentation de la prescription initiale hospitalière ou de la prescription d'un autre médecin accompagnée d'une prescription initiale hospitalière datant de moins d' 1 an. Ces règles sont valables pour toutes les formes galéniques $(2,3,4)$. Enfin, le MPH est inscrit sur l'édition 2006 de la liste des substances et méthodes interdites en compétition du Code Mondial Antidopage (28).

\section{Contre-indications et effets secondaires}

Le MPH est contre-indiqué en cas de manifestations d'angoisse ou de manifestations psychotiques, d'antécédents personnels et/ou familiaux de tics moteurs ou de maladie de Gilles de la Tourette. Il est également contre-indiqué en cas de glaucome, d'hyperthyrö̈die, d'affections cardio-vasculaires sévères, chez l'enfant de moins de 6 ans et chez les jeunes filles en âge de procréer. Enfin, il ne doit pas être administré pendant la grossesse et l'allaitement (15).

Les effets indésirables à court terme sont généralement mineurs, transitoires, et essentiellement observés pour des posologies élevées. Il peut s'agir de troubles du sommeil, d'anxiété, d'instabilité thymique, d'anorexie, ou encore de vertiges. Ces troubles disparaissent généralement spontanément après quelques semaines de traitement ou après réduction de la posologie (15).

Les effets indésirables à moyen et long terme sont représentés par les troubles de la croissance et les conduites addictives. Le MPH ralentit la croissance en période de traitement ; l'interruption du traitement en fin de semaine et pendant les vacances chez les enfants prépubères est associée à une croissance compensatrice avec normalisation de la taille qui n'est donc pas compromise en fin d'adolescence (15). Le potentiel addictif fera l'objet d'un chapitre à part entière.

\section{Potentiel addictif et cas médico-légaux.}

Le potentiel addictif du MPH est un sujet très fortement soumis à polémique, les auteurs français tendant à minimiser ce problème par rapport aux auteurs anglophones, américains en particulier. Ce phénomène peut être expliqué par le fait que la prescription de MPH en France est soumise à des indications plus restreintes ainsi qu'à des contraintes légales beaucoup plus sévères limitant considérablement son accès.

Il semble en fait nécessaire de distinguer deux types de comportements différents : l'usage dans un contexte récréatif, le plus souvent dans une population étudiante qui se procure le produit sur le marché clandestin, et le 
détournement d'usage chez des adolescents ou des adultes traités pour un THADA. Aucun cas de mésusage n'a été décrit chez l'enfant.

L'usage de MPH dans un but récréatif est un phénomène qui se développe rapidement aux États-Unis dans le milieu étudiant (29 - 32). Selon Babcok et al. (30), cet usage semble aussi fréquent dans les universités que celui des amphétamines ou de la cocaïne. Les étudiants utilisent le MPH soit dans un contexte festif en association avec l'alcool et le cannabis, soit pour augmenter leurs capacités d'attention et d'éveil dans le cadre de leurs études (32). Dans ce contexte d'usage, les overdoses sont possibles mais très rares. Massello et Carpenter (33) décrivent ainsi un cas de décès par surdosage chez un adolescent ayant absorbé du MPH dans un but récréatif. Les principales voies d'absorption sont la voie orale et la voie nasale après pulvérisation (34). Les doses absorbées sont très variables, la dose la plus élevée mentionnée dans la littérature étant de $200 \mathrm{mg}$ (35). Il semblerait d'autre part que les utilisateurs récréatifs soient plus facilement susceptibles de déclarer un usage intranasal ainsi qu'une co-administration avec d'autres substances, alors que l'absorption par voie orale semble préférée lorsqu'il s'agit d'augmenter les performances intellectuelles $(30,32)$. De manière générale, les usagers de MPH déclarent avoir utilisé au cours de leur vie d'autres stimulants, licites ou illicites, beaucoup plus souvent que les sujets témoins (32). Selon Weiner (36), la diffusion de ce phénomène est lié à la facilité d'obtention du produit qui circule de manière illicite sous des appellations diverses : «Vitamin R », «Skippy », « The smart Drug », « R-Ball » $(34,37)$. Selon la Drug Enforcement Agency (38), le MPH fait ainsi l'objet d'un véritable marché clandestin aux États-Unis.

A côté de cet usage récréatif, d'authentiques cas d'abus et de dépendance - selon les critères du DSM-IV - au MPH sont décrits dans la littérature chez des adolescents ou des adultes traités pour un THADA $(35,39$, 40). Il arrive également que l'abuseur ne soit pas le patient traité mais un membre de son entourage. Parran et al. (41) rapportent ainsi une série de 22 patients ayant présenté un comportement d'abus, 9 d'entre eux ayant des enfants traités par une forme de MPH à libération prolongée. Fulton et al. (42) décrivent même un cas d'abus familial.... Dans ces cas, la voie d'administration est généralement nasale ou intraveineuse, après pulvérisation et dissolution dans l'eau (34). La voie intraveineuse semble être l'apanage des adultes (34). Les doses sont variables, de 40 à $1000 \mathrm{mg}$ (43). Des complications à type d'abcès, de cellulite, de sclérose veineuse et d'embolies artérielles ont été décrites chez ces usagers (41). Un cas d'overdose après injection intraveineuse de $40 \mathrm{mg}$ de MPH (44) a été décrit; les concentrations retrouvées étaient de $2,8 \mathrm{mg} / \mathrm{l}$ dans le sang, $5,7 \mathrm{mg} / \mathrm{l}$ dans la bile et $1,6 \mathrm{mg} / \mathrm{l}$ dans l'estomac. Ces cas sont probablement plus isolés mais certainement plus graves que ceux décrits dans un contexte récréatif. Il convient en fait de prendre en compte dans l'interprétation de ces comportements une notion importante : le THADA constitue lui-même un facteur de risque de mésusage à l'adolescence et à l'âge adulte (45 - 48), en particulier dans les formes cliniques associant des troubles du comportement (49). Les patients sont généralement des polyconsommateurs, mais pas forcément de psychostimulants, l'alcool et le cannabis étant les produits les plus fréquemment cités (50). D'autre part, de nombreuses équipes s'accordent à reconnaître que les psychostimulants correctement prescrits auraient plutôt un effet protecteur contre les comportements d'abus dans le cadre du THADA (51, 52).

En France, l'usage récréatif du MPH semble inconnu et les cas d'abus déclarés sont extrêmement rares. Le Centre d'Evaluation et d'Information sur la Pharmacodépendance (CEIP) de Nancy déclarait dans son bulletin de décembre 2002 un cas de détournement d'usage (53). La Lettre du CEIP de Montpellier de novembre 2003 faisait également état d'un abus de Ritaline $^{\oplus}$ et d'Effexor ${ }^{\oplus}$ chez un patient de 43 ans (54). Dans ce dernier cas, le patient a signalé que les pharmaciens lui ont délivré le produit sans présentation de la prescription initiale hospitalière. Une observation personnelle (55) relève le même dysfonctionnement. Ces observations tendent à démontrer que le dispositif réglementaire français, lorsqu'il est appliqué correctement, est efficace pour limiter ces comportements d'abus.

Les effets recherchés par les usagers sont comparables à ceux des autres stimulants. Selon Volkow et al. (56), l'absorption de MPH par voie nasale provoque des effets similaires à ceux de l'amphétamine ou du crack, et selon Wang et al. (57), l'administration par voie intraveineuse a des effets similaires à ceux d'une administration intraveineuse de cocaïne. Ces manifestations sont caractérisés par un effet « high » très rapide, suivi d'une euphorie intense, d'une sensation d'hypervigilance, d'hyperactivité et d'anorexie pendant une durée d'environ 6 heures $(39,56)$. La tolérance apparaît rapidement. Le syndrome de manque, proche de celui observé avec les amphétamines, est caractérisé par une dysphorie, une asthénie intense, des troubles du sommeil (34). Les overdoses sont également caractérisées par des manifestations comparables à celles observées avec les autres psychostimulants (34), i.e. des manifestations neuropsychiatriques à type d'agitation, halluci- 
nations, syndrome confusionnel, convulsions et troubles de la conscience d'intensité variable, et des manifestations cardiovasculaires à type de tachycardie, hypertension, troubles du rythme auriculaires et ventriculaires. L'hyperthermie et l'arythmie sont l'apanage des formes sévères $(34,43)$.

Les mécanismes neurobiologiques de la dépendance au MPH sont mal connus. Le profil pharmacodynamique du MPH est très proche de celui des amphétamines et de la cocaine, et les effets subjectifs ressentis par les usagers sont comparables, en particulier lorsque le MPH est administré par voie intraveineuse $(43,57)$. Des études supplémentaires seront nécessaires pour affiner la connaissance de ces phénomènes.

\section{Aspects analytiques}

L'extraction du MPH et de l'acide ritalinique sous forme racémique se fait soit en phase liquide (58 - 60), soit en phase solide sur cartouches SPE $(61,62)$. Les premières techniques séparatives développées pour le dosage du MPH et de l'acide ritalinique sous forme racémique dans le plasma et les urines faisaient appel à la chromatographie sur couche mince (63), à la chromatographie en phase gazeuse couplée à un détecteur thermoionique $(62,64)$, puis à la spectrométrie de masse (CG-SM) en ionisation chimique positive ou négative $(58,65,66)$ ou electrospray (60). La limite de quantification de ces techniques se situe généralement autour de $0,4 \mathrm{ng} / \mathrm{ml}$ pour le MPH et son métabolite. Une technique d'électrophorèse capillaire couplée à la spectrométrie de masse a également été développée, mais avec une limite de quantification plus élevée, de l'ordre de $1,5 \mathrm{ng} / \mathrm{ml}$ (67). Des techniques ELISA ont été mises au point pour le screening urinaire (68-69), mais avec parfois un taux élevé de faux positifs (69). Actuellement, la majorité des auteurs s'accordent à reconnaître la supériorité de la chromatographie liquide couplée à la spectrométrie de masse (CL-SM), et surtout à la spectrométrie de masse en tandem (CL-SM-SM) (61, 69, 70). Ramos et al. (70) décrivent une technique de dosage dans le plasma avec ionisation par source APCI, acquisition en mode MRM (transition de quantification $234.5 \rightarrow 83.9$ ), la limite de quantification est à $50 \mathrm{pg} / \mathrm{ml}$. Eichhorst et al. (69) réalisent le dosage du MPH et de l'acide ritalinique dans les urines en utilisant une source de type ionspray à pression atmosphérique en mode ionisation positive. L'acquisition se fait en mode SRM, les transitions étant respectivement $234.3 \rightarrow 84.9$ et $220.3 \rightarrow 84.3$ pour le MPH et l'acide ritalinique. La séparation et la quantification des énantiomères peuvent être effectuées par chromatographie liquide avec détection dans l'UV
(71), CG-SM (72), CL-SM (73), CL-SM-SM (69) en utilisant des phases stationnaires chirales de type macrocycliques glycopeptidiques (69), ou AGP (71).

\section{Conclusion}

Même si potentiel addictif du MPH reste un sujet très controversé, en particulier en France, la multiplication des cas d'abus et de dépendance rapportés dans la littérature doit inciter à la plus grande prudence. Il semble néanmoins que le respect très strict des indications thérapeutiques et des règles de prescription, points sur lesquels la majorité des auteurs s'accordent, parvienne à limiter le phénomène. Il est donc souhaitable que les autorités sanitaires et les médecins prescripteurs français restent très vigilants afin de prévenir le développement d'un phénomène de plus grande ampleur à l'instar de celui observé aux États-Unis.

\section{Références}

1. Physicians' Desk Reference. Ritalin (methylphenidate), 11 th edn. Oradell NJ: Medical Economics, 1956; 441-2.

2. Ritaline $10 \mathrm{mg}$ comprimés. Commission de la Transparence. Avis du 12 juin 2002. http://afssapsprd.afssaps.fr/html/has/sgt/htm/avis/data/ct021138.pdf

3. Ritaline LP $20 \mathrm{mg}$ gélules. Commission de la Transparence. Avis du 14 janvier 2004. http://afssapsprd.afssaps.fr/html/has/sgt/htm/avis/data/ct031495.pdf

4. Concerta LP. Commission de la Transparence. Avis du 29 octobre 2003. http://afssaps-prd.afssaps.fr/html/has/ sgt/htm/avis/data/ct031455.pdf

5. Mission Interministérielle de Lutte contre la Drogue et la Toxicomanie. Revue de presse du 23 novembre 2005. http://www.drogues.gouv.fr/fr/index.html

6. Moffat A.C., Jackson J.V., Moss M.S., Widdop B. Clarke's isolation and identification of drugs. 2th edn. London : The Pharmaceutical Press, 1986 : 769-70.

7. Leonard B.E., McCartan D., White J., King D.J. Methylphenidate : a review of its pharmacological, neuropsychological and adverse clinical effects. Hum. Psychopharmacol. $2004 ; 19$ : 151-80.

8. Patrick K.S., Markowitz J.S. Pharmacology of methylphenidate, amphetamine enantiomers and pemoline in attention-deficit hyperactivity disorder. Hum. Psychopharmacol. 1997 ; $12: 527-46$.

9. Markowitz J.S., Straughn A.B., Patrick K.S., DeVane C.L., Pestreich L., Wang Y., Muniz R. Pharmacokinetics of methylphenidate after oral administration of two modified-release formulations in healthy adults. Clin. Pharmacokinet. $2003 ; 42$ : 393-401.

10. Midha K.K., McKay G., Rawson M.J., Korchinsky E.D., Hubbard J.W. Effects of food on the pharmacokinetics of methylphenidate. Pharm. Res. 2001 ; 18 : 1185-9. 
11. Hungund B.L., Perel J.M., Hurvic M.J., Sverd J., Winsberg B.G. Pharmacokinetics of methylphenidate in hyperkinetic children. Br. J. Clin. Pharmacol. $1979 ; 8$ : 571-6.

12. Sun Z., Murry D.J., Sanghani S.P., Davis W.I., Kedishvili N.Y., Zou Q.Z., Hurley T.D., Bosron W.F. Methylphenidate is stereoselectively hydrolysed by human carboxylesterase CES1A1. J. Pharmacol. Exp. Ther. $2004 ; 310: 469-76$.

13.Zhang J., Deng Y., Fang J., McKay G. Enantioselective analysis of ritalinic acids in biological samples by using a protein-based chiral stationary phase. Pharm. Res. 2003 ; $20: 1881-4$.

14. Markowitz J.S., DeVane C.L., Boulton D.W., Nahas Z., Rish S.C., Diamond F., Patrick K.S. Ethylphenidate formation in human subjects after the administration of a single dose of methylphenidate and ethanol. Drug Metab. Dispos. $2000 ; 28: 620-4$.

15. Bricard C., Boidein F. Prescription de Ritaline ${ }^{\circledR}$ dans le cas d'une prise en charge multimodale du trouble d'hyperactivité avec déficit de l'attention. Encéphale. $2001 ; 27$ : 435-43.

16. Srinivas N.R., Hubbard J.W., Korchinsky E.D., Midha K.K. Enantioselective pharmacokinetics of dl-threomethylphenidate in humans. Pharm. Res. 1993 ; 10 : 14-21.

17. Volkow N.D., Wang G.J., Fowler J.S., Ding Y.S. Imaging the effects of methylphenidate on brain dopamine: new model on its therapeutic actions for attentiondeficit/hyperactivity disorder. Biol. Psychiatry. 2005 ; 57 : 1410-5.

18. Mach R.H., Nader M.A., Ehrenkaufer R.L., Scott W.L., Smith C.R., Gage H.D., Morton T.E. Use of positron emission tomography to study the dynamics of psychostimulant-induced dopamine release. Pharmacol. Biochem. Behav. 1997 ; 57 : 477-86.

19. Shenker A. The mechanism of action of drugs used to treat attention-deficit hyperactivity disorder: focus on catecholamine receptor pharmacology. Adv. Pediatr. $1992 ; 39$ : 337-82.

20. Barkley R.A., Murphy J.V. Treating attention-deficit hyperactivity disorder : medication and behaviour management training. Pediatr. Ann. $1991 ; 20: 256-60$.

21. Swanson J., Gupta S., Lam A., Shoulson I., Lerner M., Modi N., Lindemulder E., Wigal S. Development of a new once-a-day formulation of methylphenidate for the treatment of attention-deficit/hyperactivity disorder: proof-ofconcept and proof-of-product studies. Arch. Gen. Psychiatry. $2003: 60: 204-11$.

22. Pelham W.E., Manos M.J., Ezzell C.E., Tresco K.E., Gnagy E.M., Hoffman M.T., Onyango A.N., Fabiano G.A., Lopez-Williams A., Wymbs B.T., Caserta D., Chronis A.M., Burrows-Maclean L., Morse G. A doseranging study of a methylphenidate transdermal system in children with ADHD. J. Am. Acad. Child Adolesc. Psychiatry. $2005 ; 44$ : 522-9.

23. Galynker I., Ieronimo C., Miner C., Rosenblum J., Vilkas N., Rosenthal R. Methylphenidate treatment of negative symptoms in patients with dementia. J. Neuropsychiatry Clin. Neurosci. $1997 ; 9: 231-9$.

24. Kittur S., Hauser P. Improvement of sleep and behavior by methylphenidate in Alzheimer's disease. Am. J. Psychiatry. $1999 ; 156: 1116-7$.
25. Bruera E., Driver L., Barnes E.A., Willey J., Shen L., Palmer J.M., Escalante C. Patient-controlled methylphenidate for the management of fatigue in patients with advanced cancer : a preliminary report. J. Clin. Oncol. 2003 ; $21: 4439-43$.

26. Hagerman R.J., Murphy M.A., Wittenberger M.D. A controlled trial of stimulant medication in children with the fragile X syndrome. Am. J. Med. Genet. 1988 ; 30 : 377-92.

27. Convention Unique sur les Stupéfiants de 1961. Liste des substances psychotropes placées sous contrôle international. http://www.incb.org/pdf/f/list/verte.pdf

28. Liste des méthodes et substances interdites en compétition pour l'année 2006. Code Mondial Antidopage. http://www.wada-ama.org/rtecontent/document/2006_ LIST_French.pdf

29. Musser C.J., Ahmann P.A., Theye F.W., Mundt P., Broste S.K., Mueller-Rizner N. Stimulant use and the potential for abuse in Wisconsin as reported by school administrators and longitudinally followed children. J. Dev. Behav. Pediatr. $1998 ; 19: 187-92$.

30. Babcock Q., Byrne T. Student perceptions of methylphenidate abuse at a public liberal arts college. J. Am. Coll. Health. $2000 ; 49: 143-5$.

31. Teter C.J., McCabe S.E., Boyd C.J., Guthrie S.K. Illicit methylphenidate use in an undergraduate student sample: prevalence and risk factors. Pharmacother. $2003 ; 23: 609-17$.

32. Barrett S.P., Darredeau C., Bordy L.E., Pihl R.O. Characteristics of methylphenidate misuse in a university student sample. Can. J. Psychiatry. 2005 ; 50 : 457-61.

33. Massello W., Carpenter D.A. A fatality due to the intranasal abuse of methylphenidate (Ritalin $($ )). J. Forensic Sci. $1999 ; 44: 2201-1$

34. Klein-Schwartz W. Abuse and toxicity of methylphenidate. Curr. Opin. Pediatr. $2002 ; 14: 219-23$.

35. Jaffe S.L. Intranasal abuse of prescribed methylphenidate by an alcohol and drug abusing adolescent with ADHD. J. Am. Acad. Adolesc. Psychiatry. $1991 ; 30$ : 773-5.

36. Weiner A.L. Emerging drugs of abuse in Connecticut. Conn. Med. $2000 ; 64: 1923$.

37. Kollins K.R., MacDonald E.K., Rush C.R. Assessing the abuse potential of methylphenidate in nonhuman and human subjects. Pharmacol. Biochem. Behav. 2001 ; 68: 611-27.

38. Drug Enforcement Agency. Congressional Testimony. 2000. http://www.usdoj.gov/dea/pubs/cngrtest/ct051600.htm

39. Garland E.J. Intranasal abuse of prescribed methylphenidate. J. Am. Acad. Child Adolesc. Psychiatry. 1998 ; 37 : $573-4$.

40. Coetzee M., Kaminer Y., Morales A. Megadose intranasal methylphenidate (Ritalin) abuse in adult attention deficit hyperactivity disorder. Subst. Abus. 2002 ; 23 : 165-9.

41. Parran T.V., Jasinski D.R. Intravenous methylphenidate abuse. Arch. Intern. Med. 1991 ; 151 : 781-3.

42. Fulton A.L., Yates W.R. Family abuse of methylphenidate. Am. Fam. Physician. $1988 ; 38: 143-5$.

43. Morton M.A., Stockton G.G. Methylphenidate abuse and psychiatric side effects. Primary Care Companion J. Clin. Psychiatry. $2000 ; 2$ : 159-64.

44. Levine B., Caplan Y.H., Kauffman G. Fatality resulting from methylphenidate overdose. J. Anal. Toxicol. 1986 ; $10: 209-10$. 
45. Shekim W.O., Asarnow R.F., Hess E., Zaucha K., Wheeler N. A clinical and demographic profil of a sample of adults with ADHD, residual state. Compr. Psychiatry. 1990 ; 31 : 416-25.

46. Disney E.R., Elkins I.J., McGue M., Iacono W.G. Effects of ADHD, conduct disorder, and gender on substance use ad abuse in adolescence. Am. J. Psychiatry. $1999 ; 156$ : 1515-21.

47. Gordon S.M., Tulak F., Troncale J. Prevalence and characteristics of adolescent patients with co-occuring $\mathrm{ADHD}$ and substance dependence. J. Addict. Dis. $2004 ; 23$ : 31-40.

48. Schubiner H. Substance abuse in patients with attention-deficit hyperactivity disorder. CNS Drugs. $2005 ; 19: 643-55$.

49. Flory K., Milich R., Lynam D.R., Leukefeld C., Clayton $R$. Relation between childhood disruptive behaviour disorders and substance use and dependence symptoms in young adulthood: individuals with symptoms of ADHD and conduct disorder are uniquely at risk. Psychol. Addict. Behav. $2003 ; 17: 151-8$.

50. Biederman J., Wilens T.E., Mick E., Milberger S., Spencer T.J., Faraone S.V. Psychoactive substance use disorders in adults with attention deficit hyperactivity disorder: effects of ADHD and psychiatric comorbidity. Am. J. Psychiatry. $1995 ; 152: 1652-8$.

51. Wilens T.E., Faraone S.V., Biederman J., Gunawardene S. Does stimulant therapy of ADHD beget later substance abuse: a meta analyse review of the literature. Pediatrics. $2003 ; 11: 179-85$.

52. Barkley R.A., Fisher M., Smallish L., Fletcher K. Does the treatment for attention-deficit/hyperactivity disorder with stimulants contribute to drug use/abuse? A 13-year prospective study. Pediatrics. $2003 ; 111: 97-109$.

53. Bulletin Régional sur la Pharmacodépendance. Bulletin $n^{\circ}$ 2. Décembre 2002. CEIP du Nord-Est. http://www.centrespharmacodependance.net/nancy/nancy1_dec_2002.pdf

54. Lettre du CEIP. Novembre 2003. http://www.centres-pharmacodependance.net/montpellier/lettre_ceip_MP_nov_20 03.pdf

55. Pelissier-Alicot A.L., Piercecchi-Marti M.D., Bartoli C., Kuhlmann E., Coiffait P.E., Sanvoisin A., Giocanti D., Leonetti G. Abusive prescription of psychostimulants : a study of two cases. J. Forensic Sci. Sous presse.

56. Volkow N.D., Ding Y.S., Fowler J.S., Wang G.J., Logan J., Gatley J.S., Dewey S., Ashby C., Liebermann J., Hitzemann R. Is methylphenidate like cocaïne? Studies on their pharmacokinetics and distribution in the human brain. Arch. Gen. Psychiatry. 1995 ; 52 : 456-63.

57. Wang G.J., Volkow N.D., Hitzemann R., Wong C., Angrist B., Burr G., Pascani K., Pappas N., Lu A., Cooper T., Liebermann J. Behavioral and cardiovascular effects of intravenous methylphenidate in normal subjects and cocaine abusers. Eur. Addict. Res. 1997 ; 3 : 49-54.

58. Leis H.J., Fauler G., Raspotnig G., Windischhofer W. Negative ion chemical ionization fort the determination of methylphenidate in human plasma by stable isotope dilution gas chromatography/mass spectrometry. J. Mass Spectrom. $2000 ; 35$ : 1100-4.

59. Ramos L., Bakhtiar R. Tse F.L.S. Liquid-liquid extraction using 96-well plate format in conjunction with liquid chromatography/tandem mass spectrometry for quantitative determination of methylphenidate (Ritalin(B) in human plasma. Rapid Commun. Mass Spectrom. 2000 : 14 : 740-5.
60. Yang Y., Kameoka J., Wachs T., Henion J.D., Carighead H.G. Quantitative mass spectrometric determination of methylphenidate concentration in urine using an electrospray ionization source integrated with a polymer microchip. Anal. Chem. $2004 ; 76: 2568-74$.

61. Wachs T., Henion J. A device for automated direct sampling and quantitation fom solid-phase sorbent extraction cards by electrospray tandem mass spectrometry. Anal. Chem. $2003 ; 75: 1769-75$.

62. Potts B.D., Martin C.A., Vore M. Gas-chromatographic quantification of methylphenidate in plasma with use of solid-phase extraction and nitrogen-sensitive detection. Clin. Chem. $1984 ; 30: 1374-7$.

63. Manno B.R., Manno J.E., Dempsey C.A. A thin layer chromatographic method for high volume screening of urine for methylphenidate abuse. J. Anal. Toxicol. 1986 ; $10: 116-9$.

64. Vu-Duc T., Vernay A. A safer methylation procedure with boron-trifluoride methanol reagent for gas chromatographic analysis of ritalinic acid in urine. J. Pharm. Biomed. Anal. 1992 ; 10 : 187-91.

65. Patrick K.S., Ellington K.R., Breese G.R., Kilts C.D. Gas chromatographic-mass spectrometric analysis of methylphenidate and p-hydroxymethylphenidate using deuterated internal standards. J. Chromatogr. $1985 ; 11: 329-38$.

66. Nakajima K., Kotaki H., Saitoh Y., Nakagawa F. Determination of methylphenidate and its main metabolite in plasma by gas chromatography-chemical ionization mass spectrometry. $1986 ; 34: 1701-8$.

67. Bach G.A., Henion J. Quantitative capillary electrophoresis-ion-trap mass spectrometry determination of methylphenidate in human urine. J. Chromatogr. B. Biomed. Sci. Appl. $1998 ; 707: 275-85$.

68. Lewis M.G., Lewis J.G., Elder P.A., Moore G.A. An enzyme-linked immunosorbent assay (ELISA) for methylphenidate (Ritalin) in urine. J. Anal. Toxicol. 2003 ; 27 : 342-5.

69. Eichhorst J., Etter M., Lepage J., Lehotay D.C. Urinary screening for methylphenidate (Ritalin) abuse: a comparison of liquid chromatography-tandem mass spectrometry, gas chromatography-mass spectrometry, and immunoassay methods. Clin. Biochem. $2004 ; 37$ : 175-83.

70. Ramos L., Bakhtiar R., Majumdar T., Hayes M., Tse F. Liquid chromatography/atmospheric pressure chemical ionization tandem mass spectrometry enantiomeric separation of dl-threo-methylphenidate, (Ritalin(B) using a macrocyclic antibiotic as the chiral selector. Rapid. Commun. Mass Spectrom. 1999 ; 13 : 2054-62.

71. Zhang J., Deng Y., Fang J., McKay G. Enantioselective analysis of ritalinic acids in biological samples by using a protein-based chiral stationary phase. Pharm. Res. 2003 ; $20: 1881-4$.

72. Lin S.N., Andrenyak D.M., Moody D.E., Foltz R.L. Enantioselective gas chromatography-negative ion chemical ionization mass spectrometry for methylphenidate in human plasma. J. Anal. Toxicol. 1999 ; 23 : 524-30.

73. Schneidermann E., Gratz S.R., Stalcup A.M. Optimization of preparative electrophoretic chiral separation of ritalin enantiomers. J. Pharm. Biomed. Anal. 2002 : $27: 639-50$. 